\title{
MATHEMATICAL MODELLING AND SIMULATION OF DELAMINATION CRACK GROWTH IN GLASS FIBER REINFORCED PLASTIC (GFRP) COMPOSITE LAMINATES
}

\author{
HASSAN IJAZ \\ University of Jeddah, Mechanical Engineering Department, Jeddah, Saudi Arabia \\ e-mail: hassan605@yahoo.com
}

\begin{abstract}
Delamination crack growth is a major source of failure in composite laminates under static and fatigue loading conditions. In the present study, damage mechanics based failure models for both static and fatigue loadings are evaluated via UMAT subroutine to study the delamination crack growth phenomenon in Glass Fiber Reinforced Plastic (GFRP) composite laminates. A static local damage model proposed by Allix and Ladevèze is modified to an non-local damage model in order to simulate the crack growth behavior due to static loading. Next, the same classical damage model is modified to simulate fatigue delamination crack growth. The finite element analysis results obtained by the proposed models are successfully compared with the available experimental data on the delamination crack growth for GFRP composite laminates.
\end{abstract}

Keywords: finite element analysis, GFRP, damage mechanics, non-local, fatigue, delamination

\section{Introduction}

Composite laminates are frequently used in modern structural materials due to the high strength-to-weight ratio. Moreover, by adjusting the orientation of fibers one can also get desired mechanical properties in desired loading directions (Herakovich, 1997). Carbon and glass fibers are commonly used to manufacture composite laminates. Carbon fibers have better strength and less density than glass fibers, but they are not cost effective. Glass Fiber Reinforced Plastic (GFRP) composite laminates are used in avionic, automobile, ship and wind turbine industries. In the present study, delamination crack growth simulations for the GFRP composite laminates are performed under static and fatigue loadings. Delamination may be defined as a crack like an entity between the composite laminates. The cracks can grow within laminates under static and fatigue loadings and may result in failure of structural parts (Davies et al., 1989; Allix and Ladevèze, 1992). Normally, damage or fracture mechanics based approaches are used to study the cark growth behavior in different structural elements. Fracture mechanics deals with the propagation of already existing crack (Meng and Wang, 2014) while, on the other hand, damage mechanics can not only simulate the propagation of cracks but also deals with initiation of the crack (Allix et al., 1995, 1998; Allix and Ladevèze, 1996; Ijaz et al., 2016).

Damage mechanics based formulations have been used to simulate the crack growth behavior in composite laminates mostly for CFRP (Corigliano, 1993; Corigliano and Allix, 2000; Chaboche et al., 1997; Alfano and Crisfield, 2001). In the present study, delamination crack growth in the GFRP composite laminates is focused using the damage mechanics based formulation.

Classical static damage models proposed by earlier authors were mostly local in nature (Allix and Ladevèze, 1992; Corigliano, 1993; Chaboche et al., 1997; Alfano and Crisfield, 2001). Localization means that damage tends to localize in a narrow zone in front of the crack tip rather than a uniform distribution over a certain region (Jirasek, 1998). Bažant and Pijaudier-Cabot 
$(1988,1989)$ proposed an integral type non-local damage model for brittle concrete materials. Similarly, a rate dependent damage model is also proposed to avoid the localization issues in CFRP composite laminates by introducing a time delay in the damage evolution formulation (Allix et al., 2000; Marguet et al., 2007). To counter the localization problem, Peerlings introduced a gradient enhanced damage evolution model (Peerlings et al., 2001). Borino gave the idea of using the integral type non-local damage model for the interface damage models for composite laminates (Borino et al., 2007). Ijaz used the idea of an integral type non-local interface damage model for the study of delamination crack growth in CFRP composite laminates (Ijaz et al., 2014). GFRP composite laminates also show a considerable amount of fiber bridging during crack growth (Davidson and Waas, 2012). In the present study, an integral type non-local damage is used to accommodate the spurious localization and fiber bridging issues during delamination crack growth in GFRP composite laminates under static loading conditions. The classical damage model proposed by Allix and Ladevèze $(1992,1996)$ is modified to a non-local one.

This article is organized as follows: in Section 2, basics of the classical interface damage model are recalled. The proposed non-local static interface damage model is discussed in Section 3. Finite element simulation results and their comparison with the experimental data are detailed in Section 4. Finally, some concluding remarks are given in Section 5.

\section{Introduction to the classical local interface damage model}

Simulation of delamination crack growth in composite laminates is performed by coupled interface damage modelling. The interface is a crack like entity that exists between two adjacent lamina layers. The relative displacement of the two adjacent layers with respect to each other can be described as

$$
\mathbf{U}=\mathbf{U}^{+}-\mathbf{U}^{-}=U_{1} \mathbf{N}_{1}+U_{2} \mathbf{N}_{2}+U_{3} \mathbf{N}_{3}
$$

where $\mathbf{N}_{1}, \mathbf{N}_{2}$ and $\mathbf{N}_{3}$ are mutual perpendicular vectors in an orthotropic reference frame for the interface. The failure or deterioration of the interface is taken into account by the introduction of three damage variables, $d_{1}, d_{2}$ and $d_{3}$ correspond to orthotropic direction vectors. Here, $d_{3}$ corresponds to the out-of-plane opening mode (Mode I), whereas $d_{1}$ and $d_{2}$ correspond to the in-plane shearing and tearing failure modes (Mode II and Mode III). The damage variable is divided into two parts, i.e. static damage variable $d_{i S}$ and fatigue damage variable $d_{i F}$. Hence, the total damage $d_{i}$ can be calculated by taking the sum of the two aforementioned damage variables $d_{i}=d_{i S}+d_{i F}, i=1,2,3$.

If $\sigma_{13}, \sigma_{23}$ and $\sigma_{33}$ are interfacial stress components in $N_{1}, N_{2}$ and $N_{3}$ directions, respectively, then the damage variables are related to the interfacial displacements as

$$
\left[\begin{array}{l}
\sigma_{13} \\
\sigma_{23} \\
\sigma_{33}
\end{array}\right]=\left[\begin{array}{ccc}
k_{1}^{0}\left(1-d_{1}\right) & 0 & 0 \\
0 & k_{2}^{0}\left(1-d_{2}\right) & 0 \\
0 & 0 & k_{3}^{0}\left(1-d_{3}\right)
\end{array}\right]\left[\begin{array}{l}
U_{1} \\
U_{2} \\
U_{3}
\end{array}\right]
$$

here $k_{1}^{0}, k_{2}^{0}$ and $k_{3}^{0}$ are defined as interface rigidities corresponding to three failure modes. The damage model is built by considering thermodynamic forces combined with damage variables and are associated with three modes of delamination as follows (Allix et al., 1995; Allix and Ladevèze, 1996)

$$
Y_{d_{3}}=\frac{1}{2} \frac{\left\langle\sigma_{33}\right\rangle_{+}^{2}}{k_{3}^{0}\left(1-d_{3}\right)^{2}} \quad Y_{d_{1}}=\frac{1}{2} \frac{\sigma_{13}^{2}}{k_{1}^{0}\left(1-d_{1}\right)^{2}} \quad Y_{d_{2}}=\frac{1}{2} \frac{\sigma_{32}^{2}}{k_{2}^{0}\left(1-d_{2}\right)^{2}}
$$


where $\left\langle\sigma_{33}\right\rangle_{+}$represents the positive value of $\sigma_{33}$, i.e. damage will not grow during compression loading when a normal loading is applied. Now the three damage variables are assumed to be strongly coupled and are governed by a single equivalent damage energy release rate of the following form (Allix and Ladevèze, 1996)

$$
\bar{Y}(t)=\max _{r \leqslant t}\left(\left(Y_{d_{3}}\right)^{\alpha}+\left(\gamma_{1} \bar{Y}_{d_{1}}\right)^{\alpha}+\left(\gamma_{2} \bar{Y}_{d_{2}}\right)^{\alpha}\right)^{\frac{1}{\alpha}}
$$

where $\gamma_{1}$ and $\gamma_{2}$ are coupling parameters, and $\alpha$ is a material parameter which governs the damage evolution under mixed mode loading conditions. Now, the damage evolution law is defined as an isotropic material function of the following form

$$
\begin{aligned}
& \text { if } \quad\left[\left(d_{3} S<1\right) \text { and }\left(\bar{Y}<Y_{R}\right)\right] \quad \text { then } \quad d_{1 S}=d_{2 S}=d_{3 S}=\omega(\bar{Y}) \\
& \text { else } \quad d_{1 S}=d_{2 S}=d_{3 S}=1
\end{aligned}
$$

where the damage evolution material function $\omega(\bar{Y})$ is defined as (Allix and Ladevèze, 1996)

$$
\omega(\bar{Y})=\left(\frac{n}{n+1} \frac{\left\langle\bar{Y}-Y_{O}\right\rangle_{+}}{Y_{C}-Y_{O}}\right)^{n}
$$

where $Y_{O}$ and $Y_{C}$ are threshold and critical damage energy release rates. $n$ is termed as a characteristic function of the material. Higher values of $n$ correspond to a brittle interface. $Y_{R}$ is defined as damage energy associated to rupture and can be calculated using following formula

$$
Y_{R}=Y_{O}+\frac{n+1}{n} d_{c}^{\frac{1}{n}}\left(Y_{C}-Y_{O}\right)
$$

Now the identification of parameters $Y_{C}, \gamma_{1}$ and $\gamma_{2}$ can be done by comparing the energy dissipation yielded by the damage mechanics approach and LEFM (Linear Elastic Fracture Mechanics). For a pure mode case energy release rate $G_{i C}(i=I, I I, I I I)$ obtained from fracture mechanics, the experiments considering LEFM can be compared to the damage mechanics approach using the following relation (Allix et al., 1995; Allix and Ladevèze, 1996)

$$
G_{I C}=Y_{C} \quad G_{I I C}=\frac{Y_{C}}{\gamma_{1}} \quad G_{I I I C}=\frac{Y_{C}}{\gamma_{2}}
$$

For a mixed-mode loading case, a standard LEFM model can be recovered as (Allix and Ladevèze, 1996)

$$
\left(\frac{G_{I}}{G_{I C}}\right)^{\alpha}+\left(\frac{G_{I I}}{G_{I I C}}\right)^{\alpha}+\left(\frac{G_{I I I}}{G_{I I I C}}\right)^{\alpha}=1
$$

The equivalence between damage mechanics and LEFM also leads to the following relation during the complete debonding process (DP)

$$
G_{I C}=\int_{D P} \sigma_{33} d U_{3} \quad G_{I I C}=\int_{D P} \sigma_{13} d U_{1} \quad G_{I I I C}=\int_{D P} \sigma_{23} d U_{2}
$$

Equation (2.9) states that for any pure mode debonding case, the area under the curve obtained from the damage mechanics approach is equal to the experimentally obtained critical energy release rate $G_{i C}$. 


\section{Non-local interface damage model for static loading}

The classical interface damage model shows the strain softening phenomenon during the degradation process (Borino et al., 2007; Ijaz et al., 2014). Due to this softening behaviour, the stress tends to localize in a narrow region in front of the crack tip. This localization phenomenon is more obvious for 3D delamination simulations over 2D analysis. Moreover, fibre bridging also occurs during delamination crack growth in GFRP composite laminates (Yao, 2015; Davidson and Waas, 2012). The mathematical non-local interface damage model presented in this Section will also inherently accommodate the fibre bridging process.

In the proposed methodology, the damage variable is made non-local by taking spatial averaging over a certain domain using the Gaussian distribution methodology. The averaging domain is dictated by the characteristic length parameter $l$. A higher value of $l$ means that more elements are used for the averaging. This domain dependent characteristic length $l$ will also simulate the fibre bridging behaviour since bridging occurs over a certain domain during delamination crack growth in composite laminates.

Now one can write the average of damage variable $d$ over the surrounding domain using the weight function $\alpha_{0}(r)$ (Ijaz et al., 2014)

$$
\bar{d}(x)=\frac{1}{V_{r}(x)} \int \alpha_{0}(\|x-\zeta\|) d(\zeta) d \zeta
$$

where

$$
V_{r}(x)=\int \alpha_{0}(\|x-\zeta\|) d \zeta
$$

The damage variable calculated using Eq. (2.5) is made non-local using Eq. (2.10) over a prescribed selected domain. The isotropic weight function $\alpha_{0}(r)$ is calculated using the Gaussian distribution function of exponential form as follows

$$
\alpha_{0}(r)=\exp \left(-\frac{r^{2}}{2 l^{2}}\right)
$$

From the above equation it is clear that the weight function $\alpha_{0}(r)$ depends on the distance between two points $r=\|x-\zeta\|$ and the characteristic length parameter $l$. A smaller value of $l$ corresponds to a less number of elements available for averaging. Higher values mean that more elements will take part in the for averaging.

Equation (2.10) describes the damage variable $d$ which is made non-local by taking spatial averaging. Similarly, like Eq. (2.10), two other variables like the equivalent damage energy release rate $\bar{Y}$ and interfacial displacement $U$ are also made non-local, and then the damage variable is calculated using these as

$$
\begin{aligned}
& \bar{Y}(x)=\frac{1}{V_{r}(x)} \int \alpha_{0}(\|x-\zeta\|) Y(\zeta) d \zeta \\
& \bar{U}(x)=\frac{1}{V_{r}(x)} \int \alpha_{0}(\|x-\zeta\|) U(\zeta) d \zeta
\end{aligned}
$$

Once the values of $\bar{Y}(x)$ and $\bar{U}(x)$ are known then the non-local damage variable can be calculated as a function of $\bar{Y}(x)$ and $\bar{U}(x)$, respectively. Once the value of the averaged non-local variable $d$ is calculated either using Eqs. (2.10) and $(2.13)_{1}$ or $(2.13)_{2}$ then the updated value of $d$ will be used in Eq. (2.2) to calculate the interfacial stresses. 


\section{Finite element analysis and results}

In this Section, details and results of finite element simulations are presented and discussed separately for both static non-local and fatigue interface damage models for GFRP composite laminates. Double Cantilever Beam (DCB) specimen for Mode I is shown in Fig. 1. The specimen has total length $L$, initial crack length $a_{0}$ and total height $2 h$, as shown in Fig. 1.

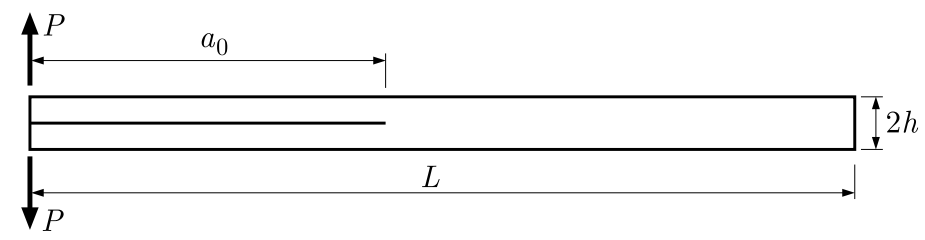

Fig. 1. DCB specimen for Mode I delamination crack growth

All simulations are performed in finite element software Cast3M (CEA) (Verpeaux et al., 2000). The geometry of the beam is modelled with 2D plane strain quadrangles. The interface between the specimen arms is modelled with 2D interface element JOI2 to simulate the debonding process (Beer, 1985). Different parameters like $Y_{O}, Y_{C}, \gamma_{1}, n, k_{1}^{0}$ and $k_{3}^{0}$ are needed to be identified for the finite element analysis of Mode I and Mode II delamination crack growth. The value of threshold damage energy $Y_{O}$ is taken zero, i.e. $Y_{O}=0$ for all the finite element simulations. The identification of $Y_{C}$ and $\gamma_{1}$ can be done by using Eq. (2.7) provided that Mode I and Mode II critical energy release rates $G_{I C}$ and $G_{I I C}$ are already determined from LEFM experiments. The identification of value of $n$ depends on the brittleness of the interface. The value of $n$ varies between $0-1.0$, and a good value can be identified by matching the experimental and numerical results. The values of interfacial rigidities can be calculated using the following relation (Ijaz et al., 2011)

$$
k_{3}^{0}=\frac{(2 n+1)^{\frac{2 n+1}{n}}}{8 n(n+1) Y_{c}} \sigma_{33}^{2} \quad k_{i}^{0}=\frac{\gamma_{i}(2 n+1)^{\frac{2 n+1}{n}}}{8 n(n+1) Y_{c}} \sigma_{3 i}^{2} \quad i=1,2
$$

In Eq. (4.1), $\sigma_{33}$ and $\sigma_{3 i}(i=1,2)$ are the maximum interfacial normal and in-plane shear stresses. The energy release rate calculated using fracture mechanics theory for pure Mode I is given as (Willams, 1988)

$$
G_{I}=\frac{M^{2}}{b E I}
$$

where $M$ is the applied moment, $b$ is width of the specimen, $E$ is flexure modulus and $I$ is the second moment of area of the bear arm.

\section{FE analysis of delamination crack growth under static loading}

In this Section, finite element analysis of Mode I delamination crack growth for GFRP composite laminates is performed using the non-local interface damage model. The experimental work of Davidson and Waas (2012) on Mode I delamination crack growth for GFRP composite laminates is used for finite element analysis. Nominal dimensions for DCB specimen are: $L=130, h=2.5$, $a_{0}=50$ and width is $b=25.4$. All the dimensions mentioned above for DCB specimen are in $\mathrm{mm}$. the Mode I critical energy release rate $G_{I C}$ value is $1.45 \mathrm{KJ} / \mathrm{m}^{2}$. The modulus $E_{11}$, in the fibre direction is $11.5 \mathrm{GPa}$ and the major Poisson's ratio is 0.3 for GFRP (Davidson and Waas, 2012). In the interface damage model, different values are identified as discussed above. The interfacial rigidity $k_{3}^{0}$ is found to be $9000 \mathrm{MPa} / \mathrm{mm}$ for maximum normal stress value of $50 \mathrm{MPa}$. Figure 2 
shows the evolution of normal stress with respect to the interfacial displacement for different identified damage parameters. From Fig. 2, one can also observe that the area under the curve is always equal to the critical energy release rate for the complete debonding process, Eq. (2.9).

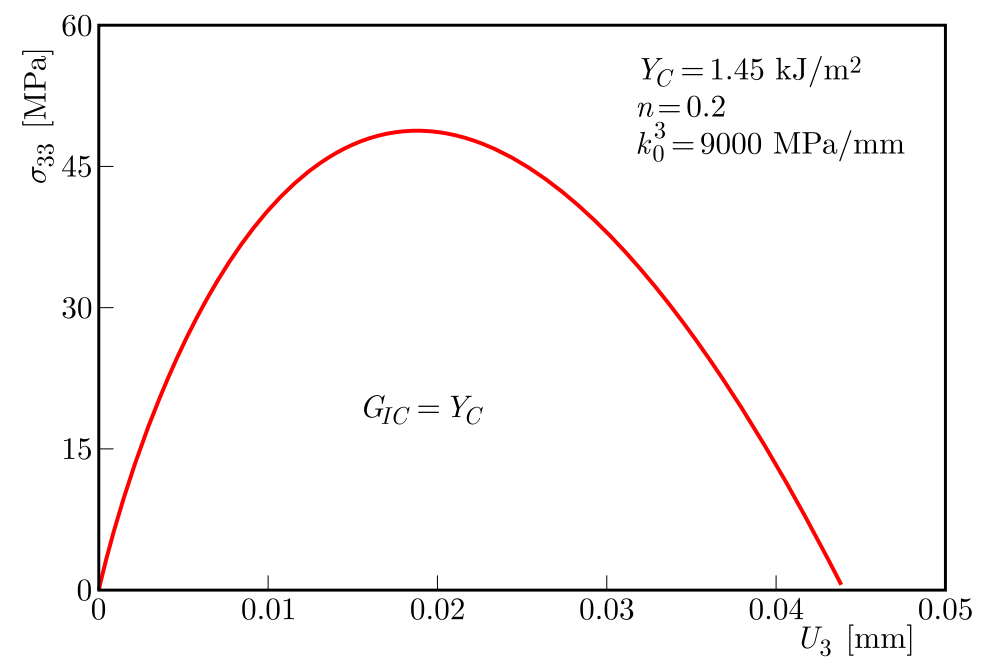

Fig. 2. Evolution of normal stress $\sigma_{33}$ with interfacial displacement $U_{3}$

Three different formulations on averaging of the variables $d, \bar{Y}$ and $U$ are discussed earlier for the non-local interface damage model. These three different non-local interface damage formulations are implemented in finite element software Cast3M via procedure PERSO1 and user material subroutine UMAT.

Figure 3 shows the normalized value of evolution of the reaction force with crack opening displacement for Mode I delamination crack growth using $d$ based non-local damage formulation. Reaction force values are normalized to $120 \mathrm{~N}$ for ease of presentation and comparison with the experimental results. Figure 3 presents the curves for the non-local model for three different characteristic lengths $l$ with values of $0.2,0.4$ and 0.6 .

(a)

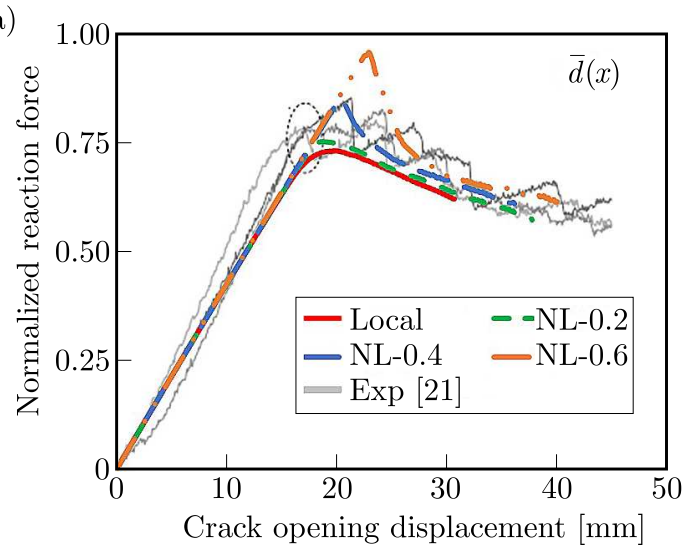

(b)

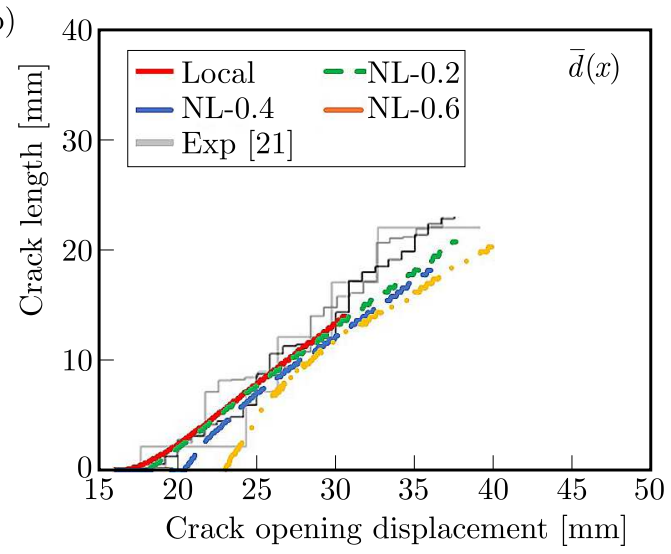

Fig. 3. (a) Normalized reaction force and (b) crack growth vs crack opening displacement for $d$ based non-local formulation

From Fig. 3a, one can notice that as the characteristic length value reduces, the results coincide with the classical local damage model proposed by Allix and Ladevèze. The reason is that as the characteristic length value reduces, a fewer number of elements is available for the averaging and the results come close to those predicted by the local damage model. The finite element simulation results are also in good agreement with the experimental results of (Davidson and Waas, 2012). Peng and Xu (2013) proposed a damage model that accommodates 
the bridging effect by dividing the damage variable into static $d_{S}$ and bridging $d_{b}$ parts. However, in the present work, the non-local model accommodates the bridging effect by controlling the characteristic length $l$. The model of Peng and Xu (2013) also demonstrated the similar behaviour of the increasing reaction force with an increase in the bridging force, whereas in the present case a larger value of the characteristic length causes an increase in the reaction force, see Fig. 3a. From Fig. 3a, one can also note that although the peak reaction force is different for various characteristic length values, but they all tend to converge close to each other once a stable crack growth is established.

Figure $3 \mathrm{~b}$ presents the evolution of delamination crack growth as a function of the crack opening displacement. Figure shows the evolution of the crack for the non-local model using three different characteristic length values, i.e. $0.2,0.4$ and 0.6. In Fig. 3, the finite element analysis results are also compared with the experimental results. The start of crack growth corresponding to different $l$ values is in accordance with the behaviour depicted in Fig. 3. The start of crack growth for the non-local damage model with a small value of $l(0.2)$ and the classical local damage model is almost the same. Similarly, crack growth starts late for a larger $l(0.6)$ value in comparison to smaller $l(0.2,0.4)$ values, and this behaviour is also in accordance with the one predicted in Fig. 3. The late start of crack growth is due to a relatively large value of $l$, which means that more elements are available for the averaging in the non-local zone and, thus, imparting extra resistance to crack growth resulting in an increase in the reaction force and, hence, the late start of crack growth. The actual fibre bridging behaviour during delamination crack growth in GFRP composite laminates is simulated by introducing the characteristic length $l$ into the classical local damage model. The larger value of $l$ indicates a wider fibre bridging zone, which means extra resistance to crack growth and a higher value of the reaction force. Moreover, there is a good agreement between numerical and experimental results for delamination crack growth with crack opening displacement, Fig. 3b.

Figure 4 shows the evolution of the reaction force and crack growth with crack opening displacement using the $U$ based non-local damage model. Trend of the reaction force and crack growth for the $U$ based non-local damage model is very similar to that predicted by $d$ based formulation. In the case of $d$ based non-local formulation, the reaction force and crack growth converge very close to each other while for $U$ based formulation both almost converge to the same path for different values of $l$ once the stable crack growth rate establishes. The results presented in Fig. 4 also show a similar trend as predicted by Peng and Xu (2013) for the reaction force in the GFRP composite laminate delamination crack growth for different bridging forces.

(a)

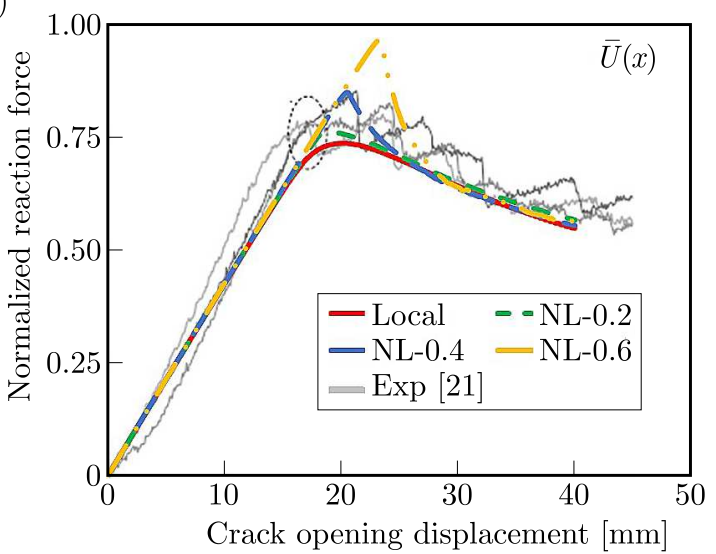

(b)

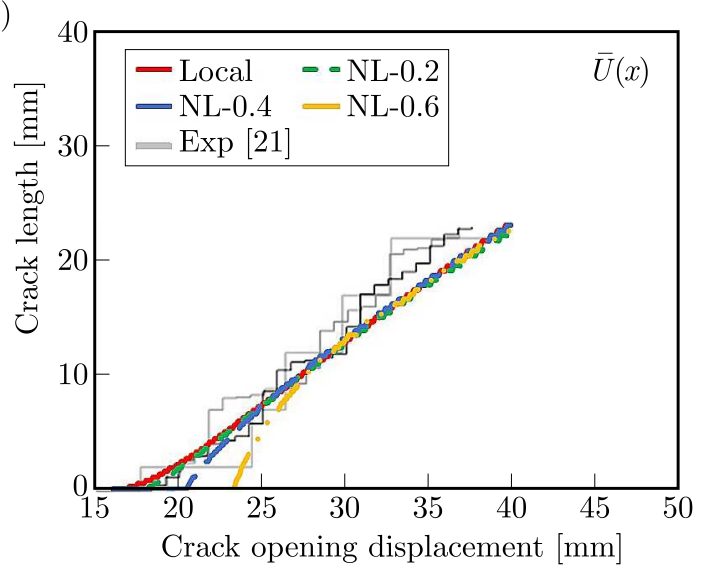

Fig. 4. (a) Normalized reaction force and (b) crack growth vs. crack opening displacement for $U$ based non-local formulation 
Similarly, Fig. 5 shows the evolution of the reaction force and crack growth with crack opening displacement using the $\bar{Y}$ based non-local damage model. The results predicted by this non-local damage model show erratic behaviour. The results start to deviate from the experimental ones as the characteristic length $l$ value increases for both the reaction force and crack growth, see Fig. 5.
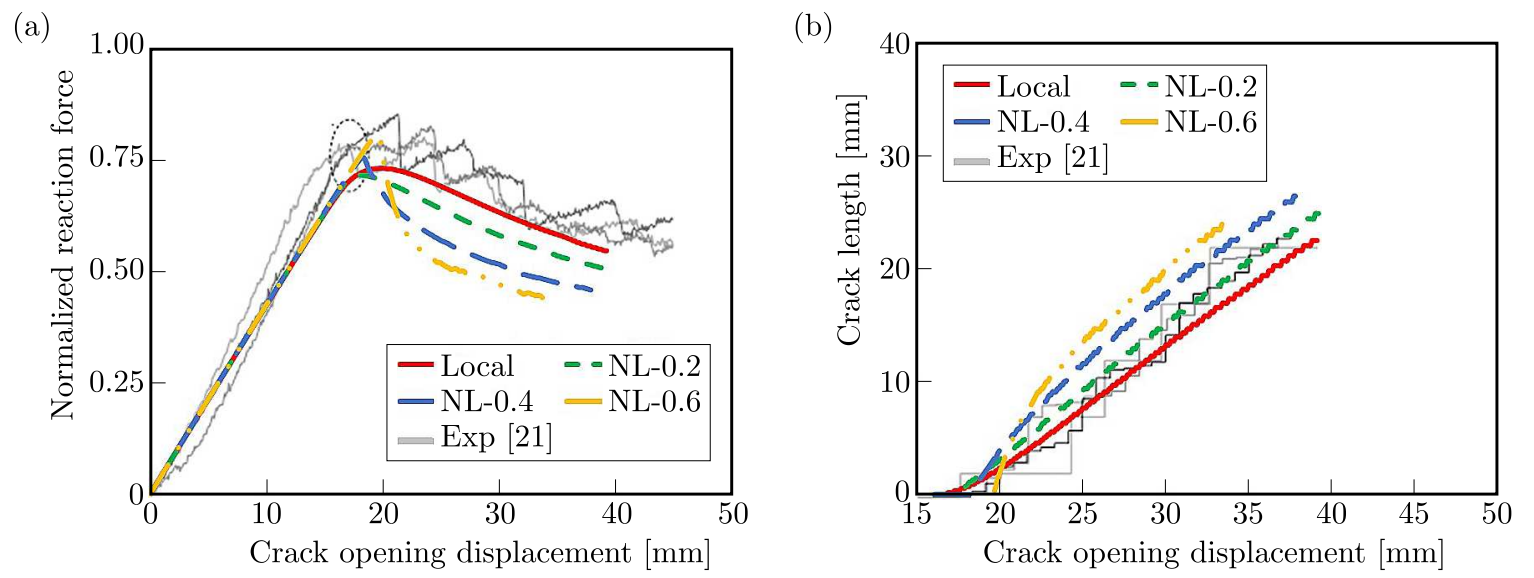

Fig. 5. (a) Normalized reaction force and (b) crack growth vs crack opening displacement for $\bar{Y}$ based non-local formulation

From the above discussions, it can be inferred that $d$ and $U$ based non-local damage formulations give reasonable results for delamination crack growths in GFRP composite laminates. The proposed methodology will not only avoid the localization issue but will also compensate the fibre bridging effect.
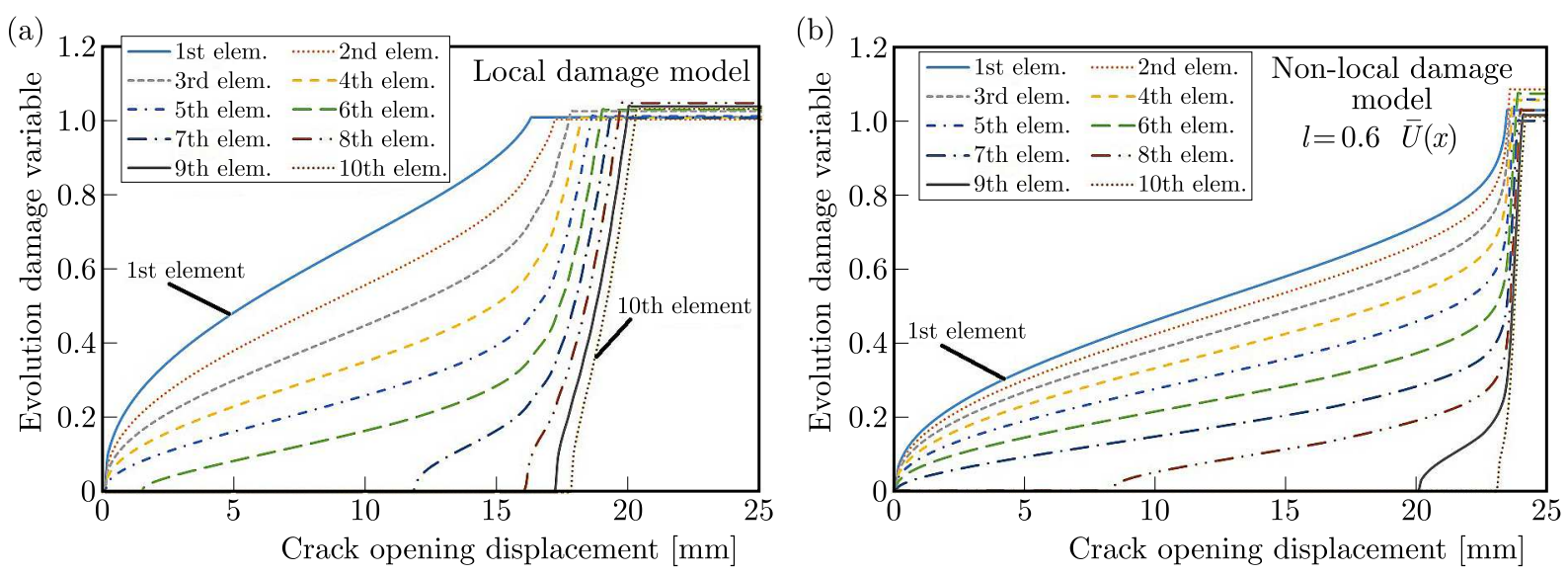

Fig. 6. Evolution of the damage variable with crack opening displacement: (a) local damage model, (b) non-local damage model, $l=0.6$

Figure 6 shows the evolution of the damage variable with crack opening displacement for the first ten elements from the crack tip for local and $U$ based non-local damage models. In the figure one can observe that for the first five elements, from the crack tip, the damage evolution starts as soon the load is applied in the local damage model, whereas in the non-local damage model, the damage evolution for the first seven elements starts as soon the load is applied. Similarly, for the eighth element, the damage evolution starts when the crack opening displacement is $17 \mathrm{~mm}$ in the local damage model, and it starts around $8 \mathrm{~mm}$ in the non-local damage model. The characteristic length $l$ helps one to involve more elements in the averaging process and makes more elements be a part of damage initiation in the non-local model in comparison to the local damage model. Hence, one can say that for larger values of $l$, more elements will be available in 
the averaging process and will take part in initial damage growth as soon as the load is applied. Since in the local damage model no averaging of damage variable is done over a certain area, therefore, fewer elements will take part in the initial damage growth in comparison with the non-local damage model, see Fig. 6.

\section{Conclusion}

Delamination crack growth in GFRP composite laminates under static loading is simulated in the present study. The original classical local damage proposed by Allix and Ladevèze is modified to a non-local static damage model and a fatigue damage model. The proposed models are implemented via UMAT subroutine and PERSO1 procedure in Cast3M FE software. The non-local damage model is not only capable of avoiding the accumulation of damage in front of the crack tip but also compensates the fibre bridging phenomenon at the interface by introducing characteristic length $l$. The reaction force and crack growth are plotted against the crack opening displacement for different values of $l$ in pure Mode I static loading. Three different formulations based on averaging of variables $d, \bar{Y}$ and $U$ are proposed. FE simulation results show that $d$ and $U$ based non-local models predict good results. The results determined by finite element analysis for the static loading is successfully compared with the available experimental data of GFRP composite laminates.

\section{References}

1. Alfano G., Crisfield M.A., 2001, Finite element interface models for the delamination analysis of laminated composites: mechanical and computational issue, International Journal for Numerical Methods in Engineering, 50, 1701-1736

2. Allix O., Ladevèze P., 1992, Interlaminar interface modelling for the prediction of delamination, Composite Structures, 22, 235-242

3. Allix O., Ladevèze P., 1996, Damage mechanics of interfacial media: basic aspects, identification and application to delamination, Studies in Applied Mechanics, 44, 167-188

4. Allix O., Ladevèze P., Corigliano A., 1995, Damage analysis of interlaminar fracture specimens, Composite Structures, 31, 61-74

5. Allix O., Ladevèze P., Deu J.F., LÉvêQue D., 2000, A mesomodel for localization and damage computation in laminates, Computer Methods in Applied Mechanics and Engineering, 183, 105-122

6. Allix O., Ladevèze P., Gornet L., Perret L., 1998, A computational damage mechanics approach for laminates: identification and comparison with experimental result, Studies in Applied Mechanics, 46, 481-500

7. Bažant Z.P., Pijaudier-Caвot G., 1988, Nonlocal continuum damage, localization instability and convergence, Journal of Applied Mechanics, 55, 287-293

8. BaŽAnt Z.P., Pijaudier-Савот G., 1989, Measurement of characteristic length of nonlocal continuum, Journal of Engineering Mechanics, 115, 755-767

9. Beer G., 1985, An isoparametric joint/interface element for finite element analysis, International Journal for Numerical Methods in Engineering, 21, 585-600

10. Borino G., Failla B. Parrinello F., 2007, Nonlocal elastic damage interface mechanical model, International Journal for Multiscale Computational Engineering, 5, 153-165

11. Chaboche J.L., Girard R., Levasseur P., 1997, On the interface debonding models, International Journal of Damage Mechanics, 6, 220-257 
12. Corigliano A., 1993, Formulation, identification and use of interface models in the numerical analysis of composite delamination, International Journal Solids and Structures, 30, 2779-2811

13. Corigliano A., Allix O., 2000, Some aspects of interlaminar degradation in composites, Computer Methods in Applied Mechanics and Engineering, 185, 203-224

14. Davidson P., WaAs A.M., 2012, Non-smooth Mode I fracture of fibre-reinforced composites: an experimental, numerical and analytical study, Philosophical Transactions of the Royal Society of London, Series A, 370, 1942-1965

15. Davies P., Cantwell W., Moulin C., Kausch, H.H., 1989, A study of delamination resistance of IM6/PEEK composites, Composites Science and Technology, 36, 153-166

16. Herakovich C.T., 1997, Mechanics of Fibrous Composites, 1st ed., John Wiley \& Sons Ltd., New York, U.S.

17. Ijaz H., Asad M., Gornet L., Alam S.Y., 2014, Prediction of delamination crack growth in carbon/fiber epoxy composite laminates using non-local interface damage model, Mechanics \& Industry, 15, 293-300.

18. IJaz H., Khan M.A., Saleem W., Chaudry S.R., 2011, Numerical modeling and simulation of delamination crack growth in cf/epoxy composite laminates under cyclic loading using cohesive zone model, Advanced Materials Research, 326, 37-52

19. Ijaz H., Zain-ul-Abdein M., Saleem W., Asad M., Mabrouki T., 2016, A numerical approach on parametric sensitivity analysis for an aeronautic aluminium alloy turning process, Mechanics, 2, 149-155

20. JIRASEK M., 1998, Nonlocal models for damage and fracture: comparison of approaches, International Journal of Solids and Structures, 35, 4133-4145

21. Marguet S., Rozycki P., Gornet L., 2007, A rate dependent constitutive model for carbon-fiber reinforced plastic woven fabric, Mechanics of Advanced Materials and Structures, 14, 619-631

22. Meng Q., Wang Z., 2014, Extended finite element method for power-law creep crack growth, Engineering Fracture Mechanics, 127, 148-160

23. Peerlings R.H.J., Geers M.G.D., De Borst R., Brekelmans W.A.M., 2001, A critical comparison of nonlocal and gradient enhanced softening continua, International Journal of Solids Structures, 38, 7723-7746

24. Peng L., Xu J., 2013, Fatigue delamination growth of composite laminates with fiber bridging: Theory and simulation, [In:] Proceeding of 13th International Conference on Fracture (ICF13), Yu S. and Feng X.-Q. (Edit.), Beijing, China, 16-21

25. Verpeaux P., Charras T., Millard A., 1998, Castem 2000: Une Approche Moderne du Calcul des Structures, Fouet J.M., Ladevèze P., Ohayon R. (Edit.), 227-261

26. Williams J.G., 1988, On the calculation of energy release rates for cracked laminates, International Journal of Fracture, 36, 101-119

27. YAO L., 2015, Mode I fatigue delamination growth in composite laminates with fibre bridging, PhD Dissertation, Technische Universiteit Delft, Germany 